\title{
Extraction of Atrial and Pulmonary Thrombi Using Angiovac Aspiration System with Transesophageal Echocardiography and Fluoroscopic Guidance
}

\author{
Saravanan Ramamoorthy ${ }^{1 *}$, Saifeldin Ahmed Mahmoud1, Kane High² \\ ${ }^{1}$ Einstein Healthcare Network, Department of Anesthesiology, Philadelphia, PA, USA \\ ${ }^{2}$ Penn State Hershey Medical Center, Department of Anesthesiology, Hershey, PA, USA \\ Email: ^RamamooS@einstein.edu, mahmouds@einstein.edu, khigh@hmc.psu.edu
}

How to cite this paper: Ramamoorthy, S., Mahmoud, S.A. and High, K. (2017) Extraction of Atrial and Pulmonary Thrombi Using Angiovac Aspiration System with Transesophageal Echocardiography and Fluoroscopic Guidance. Open Journal of Anesthesiology, 7, 160-165.

https://doi.org/10.4236/ojanes.2017.76017

Received: May 11, 2017

Accepted: June 24, 2017

Published: June 27, 2017

Copyright $\odot 2017$ by authors and Scientific Research Publishing Inc. This work is licensed under the Creative Commons Attribution International License (CC BY 4.0).

http://creativecommons.org/licenses/by/4.0/

\begin{abstract}
Surgical embolectomy is widely used to treat massive or submassive pulmonary thromboembolism and chronic thromboembolic pulmonary hypertension [1]-[3]. However, recently a new minimally invasive transvenous approach to clot evacuation using the AngioVac aspiration system (Vortex Medical, Norwell, Massachusetts) such as AngioVac aspiration system has been successfully used for retrieval of right atrial clot [4]-[6]. This is the first case report describing evacuation of trans-septal thrombus using an AngioVac aspiration system utilizing transesophageal echocardiograph (TEE) and fluoroscopic guidance. This case report also highlights the potential complications involved in AngioVac system which were readily diagnosed using TEE. We present a 66-year-old Caucasian female with a right atrial thrombus extending into left atrium through a patent for ovale (PFO) defect and a pulmonary artery thrombus. She underwent suction evacuation with an AngioVac aspiration system under TEE and fluoroscopic guidance. The right atrial thrombus extending into left atrium through a PFO was successfully evacuated through the PFO. However, the evacuation of the clot in the pulmonary artery was complicated by a rupture of the right ventricle requiring emergent sternotomy, cardiopulmonary bypass (CPB) and transient extracorporeal mechanical oxygenation (ECMO) support. The patient rapidly improved post-operatively and was discharged from hospital. Echocardiographic documentation of right heart thrombus and pulmonary emboli has poor prognostic implications. This report delineates the utility of TEE for visualization of cannula placement and real time aspiration of clots through the cannula. Potential complications associated with this technique are mechanical dislodge of thrombus from its attachment with subsequent embolization and rupture of cardiac chambers.
\end{abstract}


Accessing the thrombus in the PA can be challenging and actual visualization of the cannula in the PA is often difficult and available only through TEE. This report highlights the utility of TEE for demonstrating trans-septal clot and the importance of precise positioning of cannula in avoiding complications. In spite of complications, AngioVac aspiration system under TEE and fluoroscopic guidance may become a safe alternative for surgical removal of intracardiac thrombi.

\section{Keywords}

Cardiac Anesthesiology

\section{Background}

The current therapeutic methodologies for right atrial thrombus are associated with high mortality [1] [2]. However, the morbidity and mortality from minimally invasive evacuation system are unknown. In the recent years, new technologies have been developed for a safe evacuation of right atrial thrombus. AngioVac aspiration system (Vortex, MA) has been successfully used for retrieval of right atrial clots [3] [4] [5] [6]. However, removal of most intracardiac transseptal atrial masses often requires surgical excision. Based on current literature review, this is a first case report describing evacuation of paradoxical embolus using an AngioVac aspiration system under TEE and fluoroscopic guidance [3] [4] [5] [6] [7]. This case report also highlights the potential complications involved in minimally invasive evacuation system. Furthermore, this case report highlights the role of TEE guidance in identifying and evacuation of tran-septal clots through interatrial septal defects and its role during complication from the procedure.

We present a 66-year-old female with a right atrial thrombus extending into left atrium through an interatrial septum defect and pulmonary artery thrombus, who underwent suction evacuation with an AngioVac aspiration system under transesophageal echocardiography (TEE) and fluoroscopic guidance. The right atrial thrombus extending into left atrium through an interatrial septal defect was successfully evacuated. A small previously un-identified PFO was confirmed with TEE using color doppler and a bubble study. However, the evacuation of clot in the pulmonary artery was complicated by a rupture of right ventricle requiring emergent sternotomy, cardiopulmonary bypass (CPB) and extracorporeal mechanical oxygenation (ECMO) support.

\section{Case Presentation}

A 66-year-old female with a history of previous stroke with residual left hemiparesis presented to a tertiary academic hospital with a history of dyspnea and chest pressure. A transthoracic echocardiogram showed a right atrial thrombus. Patient's left ventricular ejection fraction was estimated to be $70 \%$. A compute- 
rized tomography scan showed left and right main pulmonary arterithrombi. The patient was also found to have deep vein thrombosis involving the right distal femoral and popliteal veins. A heparin infusion (1500 Unit per hour) was administered.

Since the patient was hemodynamically stable, a catheter based clot evacuation was deemed appropriate over open surgical embolectomy. A minimally invasive suction evacuation of the clot using AngioVac aspiration system (Vortex Medical, MA) in a hybrid operative suite was planned. After arrival in the hybrid operative suite, an awake arterial catheter was placed in the left radial artery. General anesthesia was induced with midazolam, fentanyl and rocuronium. Subsequently, a $9 \mathrm{Fr}$ introducer was placed in the left internal jugular vein. The right internal jugular vein was spared for cannulation with the AngioVac extraction device. A transesophageal echocardiography probe was placed without any complication. Initial TEE images showed an extensive thrombus in the right atrium with transient contact with the tricuspid valve extending into the left atrium through a suspected interatrial septal defect (Figure 1). In addition, a thrombus was noted in the main pulmonary artery and right pulmonary artery respectively. Right ventricular dysfunction was noted with a TAPSE measurement of $10 \mathrm{~mm}$ at the tricuspid annulus.

The right subclavian vein was accessed for insertion of AngioVac extraction cannula ( $25 \mathrm{~F}$ with balloon actuated and funnel shaped tip) and the right femoral vein was cannulated with reinfusion cannula (17 Fr) for return flow (Figure 2). The AngioVac system also included a centrifugal pump, console (Maquet, NJ), standard extracorporeal bypass tubing and filter. The extraction system was inserted into the right atrium under direct fluoroscopic and TEE visualization. The pump circuit was connected to the AngioVac cannula and reinfusion cannula after priming with warm saline solution. The luminal air was cleared and suction of clots was begun. The right atrium was cleared of clot. The clot was visualized returning through an interatrial septal defect without suggestion of embolization. A small previously unidentified PFO was confirmed with TEE using color Doppler and a bubble study (Figure 3). Multiple passes were made through the superior vena cava and the aright atrium with the AngioVac cannula at 4000 $\mathrm{rpm}(4 \mathrm{~L} / \mathrm{min})$. The AngioVac cannula was removed and the left pulmonary artery was accessed under fluoroscopic and TEE guidance. The suction of clot was begun and the clot was successfully evacuated (FigC). The AngioVac cannula was removed again and access of right pulmonary artery was started under fluoroscopic and TEE guidance. During the insertion of AngioVac cannula in the right pulmonary artery, the leading edge of the cannula was seen to flare outward into the right ventricle. At this time, the pressure tracing was lost on the left radial artery catheter. Subsequently, TEEdemonstrated fluid around the heart, and a ventricular perforation was suspected. A decision was made to institute cardiopulmonary bypass (CPB) immediately through femoral-femoral cannulation. At this time, the patient became hypotensive with mean arterial pressure at $35 \mathrm{mmHg}$. Immediate resuscitation began with administration of 


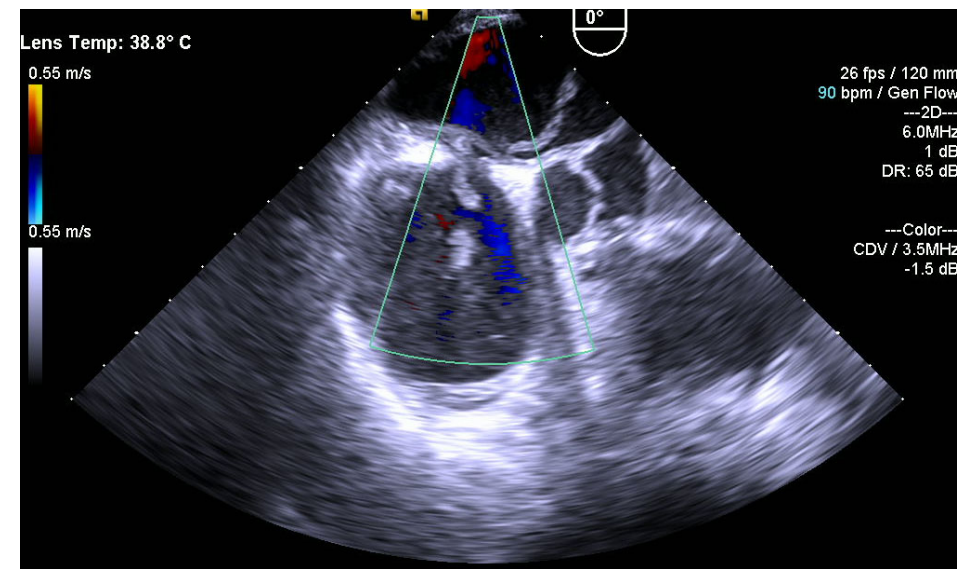

Figure 1. Shows the right atrial thrombus extending into left atrium through a potential inter-atrial defect.

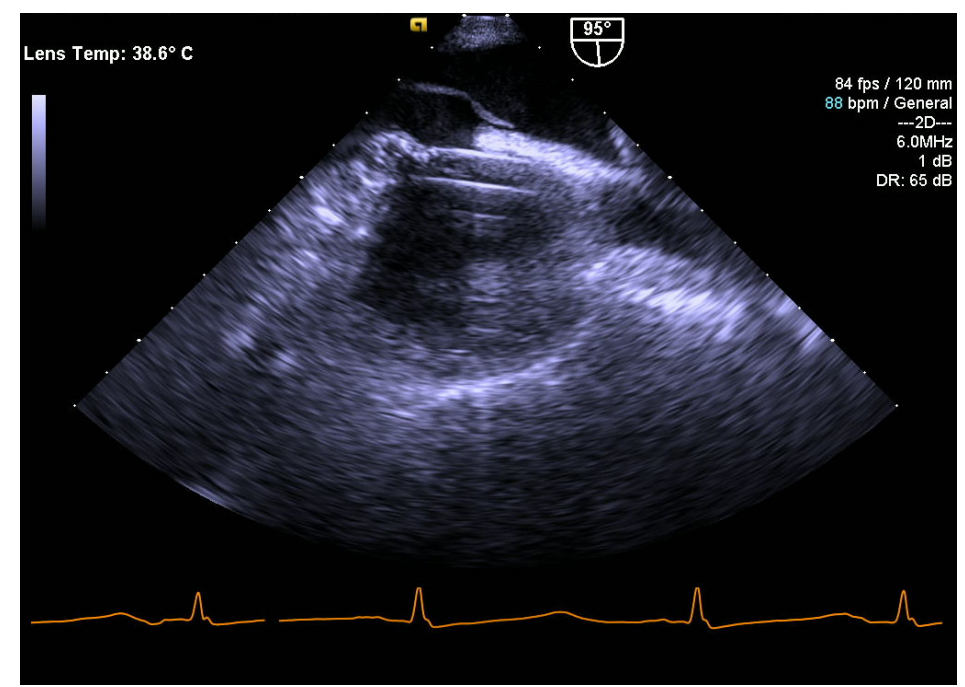

Figure 2. Shows the AngioVac aspiration system in place in $\mathrm{R}$ atrium. The right atrial clot extending into the left atrium is no longer present.

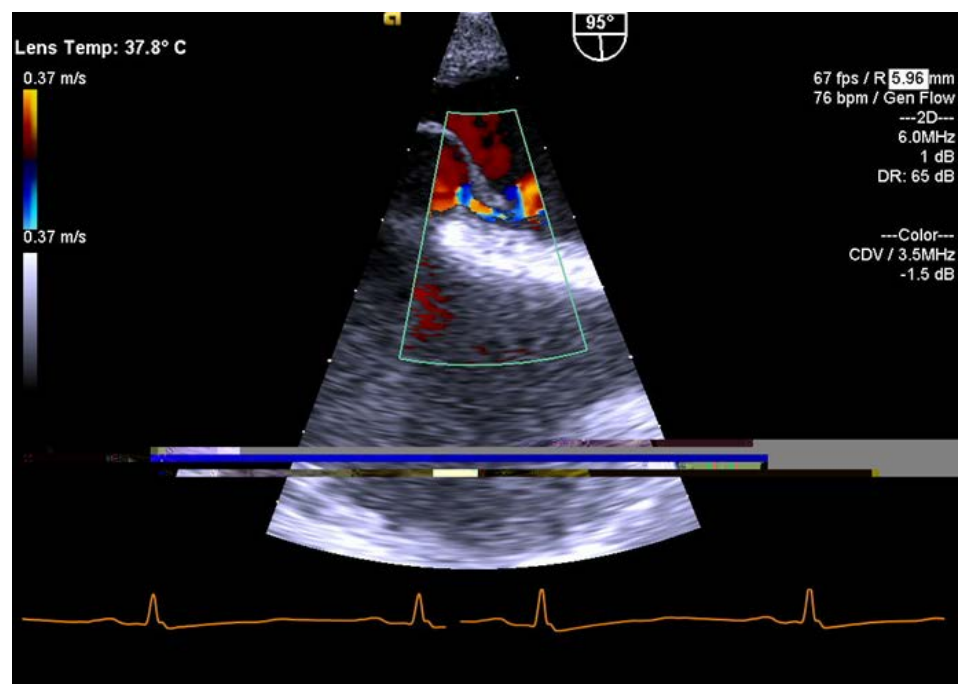

Figure 3. Shows the Color doppler with flow going through the Patent foramen ovale (PFO) subsequent to removal of the trans-atrial clot. 
epinephrine IV ( $3 \times 1 \mathrm{mg}$ bolus $)$ and establishment of rapid infusion system connected to the central line. Immediately, patient was infused with 9 units of packed red blood cells. A full dose anticoagulation with intravenous Heparin (30,000 Units) was administered. Subsequently, a femoral-femoral CPB was initiated and emergent sternotomy was performed. Upon entering into the chest cavity, a clear laceration in the right ventricle was noted. Hypothermia to $28^{\circ} \mathrm{C}$ was induced for neurological protection. The laceration in the right ventricle was repaired and further, the clot in the right pulmonary artery was evacuated. As the TEE exam noted right ventricular dysfunction with TAPSE measurement of $4 \mathrm{~mm}$, and the proximity of rupture to LAD distribution, a decision was made to wean of CPB with extra-corporeal mechanical oxygenation (ECMO) support. An epinephrine infusion of $0.05 \mathrm{mcg} / \mathrm{kg} / \mathrm{min}$ was initiated and heparin was neutralized with intravenous protamine administration $(300 \mathrm{mg})$. Subsequently, the patient was weaned off from CPB to ECMO (flow at $4 \mathrm{~L} / \mathrm{min}$ ).

The patient tolerated ECMO with minimal inotropic support (epinephrine infusion at $0.03 \mathrm{mcg} / \mathrm{kg} / \mathrm{min}$ ) and intravenous sedation with midazolam and was returned to the cardiac intensive care unit in a stable condition. The patient was initiated on hypothermic protocol at 32 degrees for 24 hours and was subsequently de-cannulated from ECMO on POD\#2. After an initial slow recovery, the patient was extubated and discharged to a rehabilitation center with return to baseline function.

\section{Discussion}

Echocardiographic documentation of right heart thrombus has a poor prognostic implication [1] [2] [3]. As in this patient, pulmonary emboli occur in $67 \%$ of patient and the mortality rate is $42 \%$ in such patients. Optimal treatment is controversial and there are no randomized trials for guidance. In majority of cases, surgical embolectomy may be preferred as it is a definitive treatment (2). Recently, however, percutaneous retrieval of thrombus without any trans-septal extension has been successfully carried out [1] [3] [8]. As per literature search, this case report, describing the evacuation of interatrial thrombus extending through a PFO using an AngioVac system, provides an alternative approach for trans-septal thrombus retrieval [1] [8]. AngioVac aspiration system may be a safe alternative for retrieval of tran-septal thrombus and evacuation of pulmonary thrombus over open surgical procedure [4] [5] [6]. Precise positioning of the AngioVac cannula is important for avoiding any complication [3]. This case report delineates the utility of TEE guidance for visualization of placement of cannula and real time aspiration of clots through the cannula. There is significant potential for complication including mechanical dislodge of the thrombus from its attachment, resulting in embolization with catastrophic hemodynamic consequences and also rupture of cardiac chambers as highlighted in this case report. Especially, accessing the thrombus in the pulmonary artery can be challenging and actual visualization of the cannula in the pulmonary artery is often unpredictable and available only through TEE. 
Further, this report highlights the utility of TEE for demonstrating trans-septal clot and the discovery of the PFO, which likely played a role in this patient's previous stroke. In spite of complications described in this report, AngioVac aspiration system under TEE and fluoroscopic guidance may become a safe alternative for surgical removal of intracardiac thrombi.

\section{References}

[1] Digonnet, A., Moya-Plana, A., Aubert, S., et al. (2007) Acute Pulmonary Embolism, a Current Surgical Approach. Interactive Cardiovascular and Thoracic Surgery, 6, 27-29. https://doi.org/10.1510/icvts.2006.141366

[2] Sekhri, V., Mehta, N., Rawat, N., Lehrman, S.G. and Aronow, W.S. (2012) Management of Massive and Nonmassive Pulmonary Embolism. Archives of Medical Science (AMS), 8, 957-969.

[3] Mukharji, J. and Peterson, J.E. (2000) Percutaneous Removal of a Large Mobile Right Atrial Thrombus Using a Basket Retrieval Device. Catheterization and Cardiovascular Interventions, 51, 479-482. https://doi.org/10.1002/1522-726X(200012)51:4<479::AID-CCD23>3.0.CO;2-F

[4] Todoran, T.M., Sobieszczyk, P.S., Levy, M.S., Perry, T.E., Shook, D.C., Kinlay, S., Davidson, M.J. and Eisenhauer, A.C. (2011) Percutaneous Extraction of Right Atrial Mass Using the AngioVac Aspiration System. Journal of Vascular and Interventional Radiology (JVIR), 22, 1345-1347. https://doi.org/10.1016/j.jvir.2011.04.004

[5] Pasha, A.K., Elder, M.D., Khurram, D., Snyder, B.A. and Movahed, M.R. (2014) Successful Management of Acute Massive Pulmonary Embolism Using AngioVac Suction Catheter Technique in a Hemodynamically Unstable Patient. Cardiovascular Revascularization Medicine, including Molecular Interventions, 15, 240-243.

[6] Divekar, A.A., Scholz, T. and Fernandez, J.D. (2013) Novel Percutaneous Transcatheter Intervention for Refractory Active Endocarditis as a Bridge to Surgery-AngioVac Aspiration System. Catheterization and Cardiovascular Interventions, Official Journal of the Society for Cardiac Angiography \& Interventions, 81, 1008-1012.

[7] Sivaram, C.A., Khastgir, T., Saneman, S.L., and Elkins, R.C. (1995) Right Atrial Mass with Attachment to the Atrial Septum Caused by Metastatic Renal Cell Carcinoma. American Heart Journal, 130, 1126-1127. https://doi.org/10.1016/0002-8703(95)90220-1

[8] Jaff, M.R., McMurtry, M.S. and Archer, S.L. (2011) Management of Massive and Submassive Pulmonary Embolism, Iliofemoral Deep Vein Thrombosis, and Chronic Thromboembolic Pulmonary Hypertension, a Scientific Statement from the American Heart Association. Circulation, 123, 1788-1830.

https://doi.org/10.1161/CIR.0b013e318214914f 
Submit or recommend next manuscript to SCIRP and we will provide best service for you:

Accepting pre-submission inquiries through Email, Facebook, LinkedIn, Twitter, etc. A wide selection of journals (inclusive of 9 subjects, more than 200 journals)

Providing 24-hour high-quality service

User-friendly online submission system

Fair and swift peer-review system

Efficient typesetting and proofreading procedure

Display of the result of downloads and visits, as well as the number of cited articles Maximum dissemination of your research work

Submit your manuscript at: http://papersubmission.scirp.org/

Or contact ojanes@scirp.org 